\title{
Desigualdades sociales en el consumo de verduras y frutas según características de los hogares argentinos
}

\author{
Social inequalities in fruit and vegetable consumption \\ by household characteristics in Argentina
}

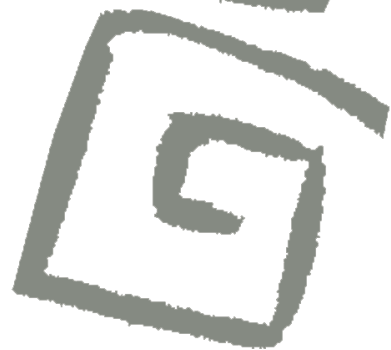

Matías Salvador Ballesteros ${ }^{1}$, María Elisa Zapata², Betina Freidin³ , Camila Tamburini ${ }^{4}$, Alicia Rovirosa ${ }^{5}$

${ }^{1}$ Doctor en Ciencias Sociales. Investigador Asistente, Consejo Nacional de Investigaciones Científicas y Técnicas, con sede en el Instituto de Investigaciones Gino Germani, Facultad de Ciencias Sociales, Universidad de Buenos Aires. Ciudad autónoma de Buenos Aires, Argentina. $\bowtie$ (iD)

${ }^{2}$ Doctora en Ciencias de la Salud. Investigadora adjunta, Centro de Estudios sobre Nutrición Infantil Dr. Alejandro O'Donnell (CESNI), Ciudad Autónoma de Buenos Aires, Argentina. $\triangle$ iD

${ }^{3}$ Doctora en Sociología. Investigadora Independiente, Consejo Nacional de Investigaciones Científicas y Técnicas con sede en el Instituto de Investigaciones Gino Germani. Universidad de Buenos Aires. Ciudad Autónoma de Buenos Aires, Argentina. $\square$ (iD)

${ }^{4}$ Licenciada en Nutrición. Investigadora Centro de Estudios sobre Nutrición Infantil Dr. Alejandro O’Donnell (CESNI), Ciudad Autónoma de Buenos Aires, Argentina. $\square$ iD

${ }^{5}$ Bioquímica. Investigadora adjunta, Centro de Estudios sobre Nutrición Infantil Dr. Alejandro O'Donnell (CESNI), Ciudad Autónoma de Buenos Aires, Argentina. $\triangle$ iD
RESUMEN El bajo consumo de verduras y frutas contribuye al aumento de la carga de enfermedades no transmisibles. Con el objetivo de analizar cómo inciden distintos factores en el consumo de verduras y frutas frescas se realizó un estudio observacional, correlacional y transversal con datos obtenidos de la Encuesta Nacional de Gastos de los Hogares 2017-2018. Se estimó el consumo aparente de verduras y frutas frescas, en gramos de peso neto. Se realizó un análisis descriptivo bivariado y uno multivariado a partir de regresiones lineales múltiples. El consumo aparente de verduras y frutas frescas está muy por debajo de lo recomendado, los menores consumos se observaron en hogares de menores ingresos, con menor clima educativo, sin adultos mayores, con menores de 14 años, con jefatura masculina y que residen en la región del nordeste argentino. Conocer las desigualdades sociales en el consumo contribuye a la planificación de políticas que garanticen la seguridad alimentaria y nutricional.

PALABRAS CLAVES Encuesta Socioeconómica; Frutas; Verduras; Factores Socioeconómicos; Argentina.

ABSTRACT Low consumption of fruits and vegetables contributes to an increased burden of non-communicable diseases. In order to analyze the impact of different factors on fresh fruit and vegetable consumption, an observational, correlational, and cross-sectional study was conducted using data collected in the 2017-2018 National Household Expenditure Survey. We calculated apparent intake of fresh fruits and vegetables in net grams. Descriptive bivariate and multivariate analyses were conducted using multiple linear regression. Apparent intake of fresh fruits and vegetables was well below the recommended level; the lowest consumption was observed in households with lower incomes, with lower educational levels, without elderly members, with children under age 14, with a male head of household, and those residing in the Argentine Northeast region. Identifying social inequalities in food consumption contributes to the development of policies aimed at promoting food and nutrition security.

KEY WORDS Socioeconomic Survey; Fruit; Vegetables; Socioeconomic Factors; Argentina. 


\section{INTRODUCCIÓN}

El objetivo de este artículo fue analizar cómo inciden distintos factores geográficos, socioeconómicos y de composición de los hogares de zonas urbanas de la Argentina en el consumo aparente de verduras y frutas a partir de los datos de la Encuesta Nacional de Gastos de Hogares (ENGHo) 2017-2018.

La alimentación es un determinante importante de la salud ${ }^{(1)}$, que desempeña un rol fundamental y afecta directamente la calidad de vida de la población ${ }^{(2)}$. La relación entre el consumo de alimentos y las enfermedades crónicas no transmisibles ha sido ampliamente estudiada en los últimos años ${ }^{(3,4,5,6)}$, y se ha estimado que una de cada cinco muertes y uno de cada seis años de vida ajustados por discapacidad se atribuyen a dietas de mala calidad $^{(7)}$.

Los cambios en la economía alimentaria mundial impactan en los hábitos y patrones alimentarios considerados determinantes de la salud ${ }^{(8,9)}$. Dentro de los cambios más importantes se advierte el descenso en el consumo de frutas y vegetales, y el aumento de alimentos con alto contenido de energía, grasas, azúcares y sodio, principalmente, ultraprocesados. Este patrón alimentario afecta negativamente la salud de la población, especialmente a los sectores sociales de menores ingresos, que además tienen una alta prevalencia de inseguridad alimentaria y de las distintas formas de malnutrición como retraso de crecimiento, deficiencia de micronutrientes, sobrepeso, obesidad, entre otras ${ }^{(10)}$.

Las frutas y verduras son componentes importantes de una dieta saludable y su bajo consumo contribuye al aumento de la carga mundial de enfermedades no transmisibles. A nivel mundial, en 2017, se atribuyeron 3,9 millones de muertes al consumo inadecuado de frutas y verduras ${ }^{(11)}$. La Organización Mundial de la Salud (OMS) y la Organización de las Naciones Unidas para la Agricultura y la Alimentación (FAO) recomiendan un consumo de frutas y verduras mayor a los 400 gramos diarios para prevenir enfermedades crónicas y deficiencias de micronutrientes ${ }^{(10)}$.
Las Guías Alimentarias para la Población Argentina (GAPA) recomiendan un consumo diario de 700 gramos, equivalentes a cinco porciones $^{(12)}$.

Con relación al estudio de la alimentación en Argentina, los datos de la ENGHo permiten observar un descenso del $27 \%$ en el consumo aparente de frutas y verduras entre 1996 y 2013, impulsado especialmente por el descenso de las frutas ${ }^{(13)}$. Esta tendencia se mantuvo en los últimos años, siendo los hogares de mayores ingresos los que tienen una dieta más variada, incluyendo diversidad de frutas y verduras ${ }^{(14)}$. La última Encuesta Nacional de Factores de Riesgo (ENFR) del año 2018 estimó que menos del $20 \%$ de la población adulta consume las cinco porciones diarias de frutas y verduras recomendadas ${ }^{(15)}$, mientras que la Encuesta Nacional de Nutrición y Salud (ENNyS) del año 2018 evidenció que solo el $33 \%$ de los mayores de dos años consume frutas al menos una vez al día y el $38 \%$ verduras, con valores significativamente menores en los grupos de niveles educativos bajos y en los de menores ingresos ${ }^{(16)}$.

El aumento de la malnutrición en todas sus formas en sectores pobres está asociado a la inseguridad alimentaria, a la mala calidad de la alimentación y a las dietas con escasa variedad de alimentos ${ }^{(17)}$. Según datos del Observatorio de la Deuda Social Argentina, la inseguridad alimentaria severa y total se registra en los grupos con mayor vulnerabilidad laboral, de ingresos y residencial ${ }^{(18)}$. Aguirre analizó las encuestas de gasto de hogares en el Área Metropolitana de Buenos Aires de los años 1965, 1970, 1985 y 1996, y observó un patrón alimentario de necesidad en los hogares pobres, con un consumo de alimentos más accesibles que "Ilenan y rinden", mientras que al ascender en la escala social predomina la elección de productos más saludables recomendados por las normativas nacionales ${ }^{(17,19)}$.

Destacamos que, en el período en que se relevaron los datos analizados en este artículo (noviembre de 2017 a noviembre de 2018), Argentina se encontraba en un importante proceso inflacionario y de deterioro de las condiciones de vida de la población, 
especialmente de los sectores más vulnerables. Según los datos del Instituto Nacional de Estadísticas y Censos (INDEC) entre diciembre de 2016 y noviembre de 2018, el índice de precios al consumidor aumentó un $78,9 \%$, mientras que el precio de los alimentos aumentó un $80,4 \%$. Las frutas lo hicieron un $68,0 \%$ y las verduras, tubérculos y legumbres un $86,1 \%{ }^{(20)}$. La inseguridad alimentaria moderada o severa en Argentina aumentó un $16,6 \%$ entre 2014-2016 y 2018-2020, pasando de $19,1 \%$ a $35,8 \%{ }^{(21)}$.

En el análisis de la problemática alimentaria argentina es importante tener en cuenta que en las últimas décadas se produjo un aumento de la producción de alimentos a partir de la consolidación del modelo de agronegocio en el marco de la globalización de la cadena agroalimentaria, lo que generó una gran pérdida en la diversidad de la producción y con alimentos de una calidad que ha sido cuestionada por los altos índices de insumos químicos utilizados. Todo ello impacta negativamente en la soberanía alimentaria ${ }^{(22)}$, y en la morbimortalidad y calidad de vida de la población debido a los elevados niveles de contaminación ambiental generada por el modelo agroalimentario dominante ${ }^{(23)}$.

\section{Perspectiva analítica}

Partimos de una perspectiva sobre las desigualdades sociales en salud en el sistema capitalista que incluye una dimensión conceptual y otra ética. Siguiendo los lineamientos de la salud colectiva latinoamericana y la epidemiología social crítica, las desigualdades derivan de la estructura de clases sociales, en su articulación con las relaciones de género y étnico-raciales que constituyen modos de vida colectivos para los distintos grupos sociales ${ }^{(24,25)}$. En el plano ético, las desigualdades son injustas y moralmente objetables cuando limitan para algunos grupos sociales el pleno goce de derechos humanos como lo son la salud y el acceso a una alimentación de calidad ${ }^{(25)}$. Siguiendo a Breilh "la salud se desarrolla como proceso concatenado entre las dimensiones espaciales de lo general, lo particular y lo singular (micro); no se puede comprender, por ejemplo, la lógica de implantación de los equipamientos, la segregación del espacio urbano, el reparto de calidades de vida entre sus barrios o zonas, los ritmos y flujos del vivir, del trabajar, del transportarse, sin entender las fuerzas y relaciones económicas del sistema de acumulación global". En el nivel microsocial se conforman "estilos de vida", a partir de patrones familiares y personales de consumo que incluyen la alimentación, que pueden ser protectores o perjudiciales para la salud ${ }^{(26)}$.

Una multiplicidad compleja de determinaciones estructurales y dinámicas socioculturales inciden en el acceso a la alimentación de calidad y en la elección de los alimentos en el ámbito del hogar. Entre ellas se encuentran las relativas al sistema de producción, suministro y comercialización; al entorno alimentario donde confluyen la calidad, la disponibilidad, la accesibilidad y la asequibilidad; y a las propias dinámicas de los individuos y grupos familiares de las que dependen los gustos, con sus hábitos, preferencias, y recursos económicos diferenciales. La capacidad de compra en el mercado, a su vez, está condicionada por las políticas públicas que inciden en los precios de los alimentos y los ingresos de los hogares, y las acciones asistenciales focalizadas ${ }^{(25,27)}$. Piaggio destaca la importancia de incluir en el debate sobre seguridad alimentaria no solo la vulneración del derecho a la alimentación adecuada por situaciones de malnutrición por déficits, sino también la producida en entornos "obesogénicos" que requieren de intervención estatal para su regulación y control. Estos entornos se caracterizan por la elevada oferta y publicidad de bebidas y alimentos ultraprocesados con contenido de grasas, azúcares y sodio ${ }^{(28)}$.

En el análisis del consumo y prácticas alimentarias inciden asimismo los roles de género, la edad, las relaciones interpersonales, la composición del hogar y aspectos geográfico-territoriales.

Son mayoritariamente las mujeres quienes asumen la responsabilidad de la alimentación del grupo familiar; el "trabajo de alimentar", y de hacerlo saludablemente, constituye una 
expectativa cultural del ejercicio de la maternidad que se transmite intergeneracionalmente y atraviesa las clases sociales ${ }^{(29)}$. En la medida en que la alimentación se conecta con la salud, el bienestar y, últimamente, con la vida, se trata de una responsabilidad de cuidado en el hogar que recae en las mujeres-madres ${ }^{(30,31,32)}$. Aunque los padres también participan de la alimentación del hogar, lo suelen hacer con un rol secundario ${ }^{(33)}$.

La edad, en tanto indicador del momento del curso de vida y de la pertenencia generacional, también condiciona las elecciones y prácticas alimentarias. Entre los adultos mayores suele ser menor la preparación de comidas elaboradas por la disminución del apetito y de la fuerza física, y por condiciones de salud propias de la edad, que Ilevan a una dieta más liviana con mayor cantidad de frutas y verduras; la simplificación de las comidas también se vincula con una menor comensalidad entre quienes viven solos debido a la viudez o separaciones ${ }^{(34,35)}$.

En cuanto a la composición del hogar, son varios los estudios que muestran cómo las mujeres-madres de niños y adolescentes "negocian" con ellos las decisiones de consumo, en gran parte por el crecimiento del mercado de productos con poco valor nutricional dirigidos a ese grupo, observándose una mayor influencia de sus preferencias ${ }^{(17,31,36)}$. Asimismo, cuando las mujeres están a cargo del hogar, sin pareja conviviente, es mayor su capacidad para decidir la composición de la dieta ${ }^{(34)}$. Por su parte, Aguirre muestra que las mujeres con ingresos propios destinan en términos porcentuales casi el doble de sus ingresos, comparado con los varones, a la compra de alimentos para el hogar ${ }^{(27)}$.

Finalmente, desde una perspectiva histórico-cultural y económica de la territorialidad, para analizar las prácticas alimentarias de los hogares es importante asimismo considerar la impronta de las cocinas regionales y locales. Con características híbridas, "son una síntesis de la cultura, de la capacidad productiva y de la historia de las regiones; son fuente de saberes y recursos culturales que forjan la identidad de las comunidades ${ }^{\prime \prime(37)}$. Respecto de los factores territoriales en el medio urbano, la oferta comercial diferencial de los distintos barrios y áreas es una dimensión del ambiente construido que afecta las posibilidades de consumo de los hogares ${ }^{(38)}$; especialmente para aquellos de menores ingresos, cuyos integrantes tienen menor capacidad de desplazamiento o traslado para satisfacer de manera deslocalizada sus necesidades cotidianas de consumo.

\section{METODOLOGÍA}

Se llevó a cabo un estudio observacional basado en datos secundarios, correlacional, y de corte transversal. Se utilizaron datos de la ENGHo realizada por el Instituto Nacional de Estadística y Censos entre noviembre de 2017 y noviembre de 2018. La muestra fue probabilística, multietápica y estratificada de 21.547 hogares de las localidades urbanas de 2.000 y más habitantes de la Argentina. Para este estudio se utilizaron las bases de datos de los cuestionarios para individuos, el hogar y los gastos diarios.

Cada hogar participante debió registrar las cantidades adquiridas de alimentos y bebidas, y el gasto correspondiente, durante el transcurso de una semana, además de las características de sus hogares e ingresos. Se consideró como consumo aparente la cantidad de alimentos y bebidas adquiridos para consumir en el hogar. A partir de los ítems del cuestionario se construyeron las variables consumo aparente de frutas frescas y consumo aparente de verduras frescas. Dentro de frutas frescas se incluyó: ananá, banana, cerezas, guindas, ciruela, damasco, durazno, frutillas, kiwi, limón, mandarina, manzana, melón, naranja, palta, pelón, pera, pomelo, sandía, uva, otras frutas frescas, surtidos de frutas frescas o congeladas. Dentro de verduras frescas se consignaron: acelga, ají, ajo, albahaca, perejil, alcauciles, apio, hinojo, arvejas, berenjenas, cebolla común, cebolla de verdeo, puerro, coliflor, brócoli, chauchas, espinaca, hongos, lechuga, pepino, rabanitos, radicheta, radicha, rúcula, remolacha, repoIlo, tomate perita, tomate redondo, tomate 
cherry, zanahoria, zapallitos, zapallo. También se incluyeron los ítems correspondientes a otras verduras y tubérculos frescos $y$ surtidos de verduras, tubérculos y legumbres frescas o congeladas, que se registran unificados en el cuestionario por lo que no es posible segmentar la categoría y separar las verduras para el análisis. En primer lugar, se realizó la conversión a cantidad de aquellos ítems registrados como unidades o atados, tomando el valor asignado por la tabla de pesos y medidas del software SARA ${ }^{(39)}$ y la tabla compilada por la Escuela de Nutrición de la Universidad de Buenos Aires ${ }^{(40)}$; en segundo lugar, para descontar las partes no consumibles del alimento (cáscaras, carozos, semillas) se aplicó a cada ítem el factor de corrección obtenido del Sistema de Análisis y Registro de Alimentos del Software SARA ${ }^{(39)}$ y de la tabla recopilada por la Escuela de Nutrición de la Universidad de Buenos Aires ${ }^{(40)}$ y se obtuvo el peso neto de cada alimento; en tercer lugar, las cantidades netas totales fueron divididas por el total de adultos equivalentes en el hogar, y transformadas a valores en gramos diarios, de forma tal que el consumo aparente se expresa en gramos de frutas y verduras frescas por adulto equivalente por día, en peso neto. Considerando que un adulto equivalente con coeficiente 1 corresponde a un varón entre 30 y 59 años, con una actividad moderada y necesidades energéticas de $2.700 \mathrm{kcal}$, cada individuo del hogar tiene su coeficiente de acuerdo a las necesidades calóricas de su grupo biológico ${ }^{(41)}$.

El análisis se realizó considerando los hogares que adquirieron alimentos y bebidas durante la semana de la encuesta.

A partir de la base de datos de individuos se construyeron las variables de composición del hogar: el sexo de la jefa o del jefe del hogar, la presencia de menores de 14 años y la presencia de adultos mayores (65 años y más). De la base de datos de hogar, se obtuvieron las variables clima educativo del hogar, quintil de ingreso per cápita, recepción de una asistencia monetaria estatal directa y ubicación geográfica (región de residencia). El clima educativo del hogar considera el promedio de años de educación de los miembros del hogar que tienen 18 años y más ${ }^{(42)}$. Se agrupó como bajo a los hogares con un promedio menor a 11 años de educación, dentro de la categoría medio a los hogares con un promedio entre 11 y menor a 16, y en la categoría alto con un promedio de 16 años y más. La asistencia monetaria estatal directa incluye la percepción de la Asignación Universal por Hijo (AUH), la Asignación Universal por Embarazo (AUE), el Programa Progresar o algún otro programa de transferencia de ingresos. Con relación a los quintiles de ingreso per cápita del hogar, el rango del 1er quintil oscila entre $\$ 0$ y $\$ 4.579$ pesos, el 2do entre $\$ 4.580$ y $\$ 7.417$ pesos, el 3ro entre $\$ 7.418$ y $\$ 10.811$ pesos, el 4 to entre $\$ 10.812$ y $\$ 17.583$ pesos y el 5 to entre $\$ 17.584$ y más (en 17 hogares, $0,1 \%$ de la muestra, declararon no haber tenido ingresos).

En primer lugar, se realizó un análisis bivariado entre cada una de las variables independientes con el consumo aparente de frutas frescas y de verduras frescas. Luego, para ambas variables dependientes se realizó un análisis multivariado a partir de una regresión lineal múltiple paso por paso agregando las variables independientes por bloques teóricos. Se utilizó el método de inclusión de variable Intro, que implica la introducción simultánea de todas las variables independientes seleccionadas en el modelo ${ }^{(43)}$. En el modelo 1 se introdujo la variable región (como variable geográfica); en el modelo 2, se agregaron las variables socioeconómicas (el quintil de ingreso per cápita del hogar, el clima educativo del hogar y la recepción de una asistencia monetaria estatal directa en el hogar); y, en el modelo 3, las variables de composición del hogar (el sexo de la jefatura del hogar, la presencia de menores de 14 años y la presencia de adultos mayores). Para realizar las regresiones se construyeron variables "dummy" y se consideró como referencia el Gran Buenos Aires para la región, el nivel alto para el clima educativo, la no recepción de una asistencia monetaria estatal directa, la jefatura masculina, la no presencia de menores de 14 años y la no presencia de adultos mayores. La variable quintil de ingreso per cápita fue introducida como cuantitativa. 
En las tablas se presenta el coeficiente de regresión lineal que indica cuántos gramos promedio diarios por adulto equivalente aumenta o disminuye el consumo aparente de frutas frescas o verduras frescas ante la presencia de cada categoría de las variables independientes (para las variables dummy) o ante el aumento de una unidad de la variable independiente (para las variables cuantitativas), una vez controlado el efecto del resto de las variables independientes consideradas en el modelo. También se incluye la significancia estadística de la prueba T de Student, que permite poner a prueba si los efectos de cada variable independiente en la muestra son estadísticamente significativos en la población. A su vez, se muestra el coeficiente de regresión estandarizado, que permite dar cuenta de la importancia relativa que tienen en el modelo las distintas variables independientes. Por último, se presenta el R cuadrado de cada modelo que indica qué parte de la variación total de cada variable de consumo aparente se debe a la variación de todas las variables independientes incorporadas en el modelo ${ }^{(43,44)}$.

Para analizar los datos se utilizaron los factores de ponderación muestral disponibles para cada hogar en la base de datos sin expandir los resultados.

\section{RESULTADOS}

\section{Caracterización de la muestra}

En la Tabla 1 se presentan las características de los 21.547 hogares de la muestra según las variables seleccionadas. Se observa que las regiones Gran Buenos Aires y Pampeana concentran dos terceras partes de la población argentina, mientras que el resto de las regiones representan el tercio restante, con los valores más bajos en la Patagonia. Con relación a las variables socioeconómicas, alrededor de uno de cada cinco hogares recibe asistencia monetaria del Estado y solo uno de cada diez hogares alcanza un clima educativo alto, es decir adultos con un promedio de al menos 16 años de educación formal. En lo
Tabla 1. Distribución porcentual de hogares de zonas urbanas, según variables seleccionadas. Argentina, 2017-2018.

\begin{tabular}{lc} 
Variables & $\%$ \\
Región & \\
GBA & 37,9 \\
Pampeana & 33,5 \\
NOA & 9,2 \\
NEA & 7,4 \\
Cuyo & 6,2 \\
Patagonia & 5,8 \\
Recepción de una asistencia monetaria estatal directa & \\
Sin asistencia & \\
\hline Con asistencia & 81,4 \\
Clima educativo del hogar & 18,6 \\
Argentino. Elaboración propia con base en la Encuesta Nacional de Gastos de \\
Bajo
\end{tabular}

que respecta a la composición del hogar, la jefatura masculina es mayor que la femenina, en el $41,5 \%$ hay niños, niñas y adolescentes menores de 14 años y en el $27,5 \%$ hay adultos mayores.

\section{Consumo de frutas frescas}

En la Tabla 2 se observa que el promedio de consumo diario de frutas frescas por adulto equivalente es de $81,5 \mathrm{~g}$. Los mayores consumos se observan en los hogares de más ingreso per cápita (134,6 g en el 5to quintil), de 
Tabla 2. Consumo aparente diario de frutas frescas por adulto equivalente (en gramos) en hogares de zonas urbanas, según variables seleccionadas. Argentina, 2017-2018.

\begin{tabular}{|c|c|}
\hline Variables & $\begin{array}{c}\text { CAD de } \\
\text { frutas frescas } \\
\text { (en gramos) }\end{array}$ \\
\hline \multicolumn{2}{|l|}{ Región } \\
\hline GBA & 86,4 \\
\hline Pampeana & 90,1 \\
\hline NOA & 70,2 \\
\hline NEA & 52,3 \\
\hline Cuyo & 74,9 \\
\hline Patagonia & 62,3 \\
\hline \multicolumn{2}{|c|}{ Recepción de una asistencia monetaria estatal directa } \\
\hline Sin asistencia & 90,7 \\
\hline Con asistencia & 41,3 \\
\hline \multicolumn{2}{|c|}{ Clima educativo del hogar } \\
\hline Alto & 131,3 \\
\hline Medio & 82,8 \\
\hline Bajo & 68,8 \\
\hline \multicolumn{2}{|c|}{ Quintil del ingreso per cápita del hogar (total del país) } \\
\hline 1er quintil & 38,6 \\
\hline 2do quintil & 56,1 \\
\hline 3er quintil & 78,1 \\
\hline 4to quintil & 100,2 \\
\hline 5to quintil & 134,6 \\
\hline \multicolumn{2}{|c|}{ Presencia de menores de 14 años en el hogar } \\
\hline No & 105,1 \\
\hline Sí & 48,2 \\
\hline \multicolumn{2}{|c|}{ Presencia de adultos mayores en el hogar } \\
\hline No & 63,3 \\
\hline Sí & 129,5 \\
\hline \multicolumn{2}{|c|}{ Sexo de la jefatura del hogar } \\
\hline Masculino & 74,9 \\
\hline Femenino & 90,3 \\
\hline
\end{tabular}

Fuente: Elaboración propia con base en la Encuesta Nacional de Gastos de Hogares 2017-2018.

$\mathrm{GBA}=\mathrm{Gran}$ Buenos Aires; NOA= Noroeste Argentino; NEA $=$ Nordeste Argentino; $C A D=$ Consumo aparente diario..

clima educativo alto $(131,3 \mathrm{~g})$, que no tienen menores entre sus integrantes $(105,1 \mathrm{~g})$ y que tienen adultos mayores $(129,5 \mathrm{~g})$, que tienen una jefatura femenina $(90,3 \mathrm{~g})$, que no reciben una asistencia estatal monetaria directa $(90,7 \mathrm{~g})$ y que residen en el GBA $(86,4 \mathrm{~g})$ o en la región Pampeana $(90,1$ g). En cambio, son los hogares de menores recursos económicos (38,6 g en el 1er quintil), con un clima educativo bajo $(68,8 \mathrm{~g})$, con menores en el hogar (48,2 g), sin adultos mayores (63,3 g), con jefatura masculina $(74,9 \mathrm{~g})$, que reciben una asistencia monetaria estatal directa $(41,3$ g), y que residen en el NEA $(52,3 \mathrm{~g})$ y en la Patagonia $(62,3 \mathrm{~g})$ los que menos fruta diaria por adulto equivalente consumen.

En la Tabla 3 se presentan los resultados de la regresión lineal múltiple para el consumo de frutas frescas. Comenzando por la variable región se observa que, en el modelo 1 , donde se encuentra como única variable los hogares del NEA, la Patagonia, el NOA, y Cuyo consumen de forma estadísticamente significativa menos frutas que los del GBA, mientras que no hay diferencias significativas con la región Pampeana. En el modelo 2, al controlar el ingreso, el clima educativo y la recepción de asistencia estatal monetaria directa, se observa que las diferencias del GBA con el NOA y con Cuyo dejan de ser significativas. Por su parte, las diferencias con el NEA continúan siendo estadísticamente significativas, pero descienden considerablemente (pasan de $-34,1 \mathrm{~g}$ a $-10,5 \mathrm{~g}$ ). Esto significa que, a igual clima educativo, la percepción de asistencia monetaria directa del Estado y el quintil de ingreso per cápita de los hogares, no son significativas las diferencias del GBA con la región de Cuyo y con el NOA, mientras que se reducen considerablemente con el NEA. Por el contrario, las diferencias con la región Pampeana se vuelven estadísticamente significativas, siendo que los hogares de esta región consumen levemente más que los del GBA. Por su parte, las diferencias con la región de la Patagonia continúan siendo significativas y de hecho aumentan levemente. Para finalizar, en el modelo 3 al controlar el efecto de las variables de composición del hogar, en NEA y en la Patagonia se continúan consumiendo significativamente menos frutas que en el GBA, mientras que no hay diferencias significativas con el resto de las regiones.

Con relación a las variables socioeconómicas de los hogares que fueron incluidas en 
Tabla 3. Regresión lineal múltiple del consumo aparente diario de frutas frescas por adulto equivalente (en gramos), en hogares de zonas urbanas. Argentina, 2017-2018.

\begin{tabular}{|c|c|c|c|c|c|c|}
\hline & \multicolumn{2}{|c|}{ Modelo 1} & \multicolumn{2}{|c|}{ Modelo 2} & \multicolumn{2}{|c|}{ Modelo 3} \\
\hline & $\begin{array}{l}\text { Coeficiente } \\
\text { de regresión } \\
\text { lineal }\end{array}$ & $\begin{array}{c}\text { Coeficiente } \\
\text { de regresión } \\
\text { estandarizado }\end{array}$ & $\begin{array}{l}\text { Coeficiente } \\
\text { de regresión } \\
\text { lineal }\end{array}$ & $\begin{array}{c}\text { Coeficiente } \\
\text { de regresión } \\
\text { estandarizado }\end{array}$ & $\begin{array}{l}\text { Coeficiente } \\
\text { de regresión } \\
\text { lineal }\end{array}$ & $\begin{array}{c}\text { Coeficiente } \\
\text { de regresión } \\
\text { estandarizado }\end{array}$ \\
\hline \multicolumn{7}{|l|}{ Región } \\
\hline GBA (referencia) & - & - & - & - & - & - \\
\hline Pampeana & 3,6 & 0,011 & $5,0^{*}$ & 0,016 & 4,0 & 0,013 \\
\hline NOA & $-16,1^{* *}$ & $-0,031$ & 2,8 & 0,005 & $-0,2$ & 0,000 \\
\hline NEA & $-34,1^{* *}$ & $-0,059$ & $-10,5^{* *}$ & $-0,018$ & $-11,9^{* *}$ & $-0,021$ \\
\hline Cuyo & $-11,5^{*}$ & $-0,018$ & $-1,1$ & $-0,002$ & $-2,9$ & $-0,005$ \\
\hline Patagonia & $-24,1^{* *}$ & $-0,038$ & $-28,6^{* *}$ & $-0,045$ & $-20,4^{* *}$ & $-0,032$ \\
\hline \multicolumn{7}{|c|}{ Recepción de una asistencia monetaria estatal directa } \\
\hline Sin asistencia (referencia) & - & - & - & - & - & - \\
\hline Con asistencia & - & - & $-15,0^{* *}$ & $-0,039$ & $-2,5$ & 0,006 \\
\hline \multicolumn{7}{|l|}{ Clima educativo del hogar } \\
\hline Alto (referencia) & - & - & - & - & - & - \\
\hline Bajo & - & - & $-18,6^{* *}$ & $-0,062$ & $-45,4^{* *}$ & $-0,151$ \\
\hline Medio & - & - & $-23,8^{* *}$ & $-0,079$ & $-33,1^{* *}$ & $-0,110$ \\
\hline \multicolumn{7}{|c|}{ Quintil de ingreso per cápita del hogar (total país) } \\
\hline Quintiles de ingreso ${ }^{1}$ & - & - & $20,9^{* *}$ & 0,197 & $14,7^{* *}$ & 0,139 \\
\hline \multicolumn{7}{|c|}{ Presencia de menores de 14 años en el hogar } \\
\hline № (referencia) & - & - & - & - & - & - \\
\hline Sí & - & - & - & - & $-22,7^{* *}$ & $-0,075$ \\
\hline \multicolumn{7}{|c|}{ Presencia de adultos mayores en el hogar } \\
\hline № (referencia) & - & - & - & - & - & - \\
\hline Sí & - & - & - & - & $54,6^{* *}$ & 0,163 \\
\hline \multicolumn{7}{|l|}{ Sexo de la jefatura del hogar } \\
\hline Masculina (referencia) & - & - & - & - & - & - \\
\hline Femenina & - & - & - & - & $13,0^{* *}$ & 0,043 \\
\hline Constante (ordenada al origen) & $86,4^{* *}$ & & $41,2^{* *}$ & & $61,9^{* *}$ & \\
\hline $\mathrm{R}^{2}$ & \multicolumn{2}{|c|}{0,006} & \multicolumn{2}{|c|}{0,056} & \multicolumn{2}{|c|}{0,092} \\
\hline
\end{tabular}

Fuente: Elaboración propia con base en la Encuesta Nacional de Gastos de Hogares 2017-2018.

Nota: Para la regresión lineal múltiple se utilizó el método de inclusión Intro y las variables independientes fueron incluidas de la siguiente forma: modelo 1 (región); modelo 2: (región, quintil de ingreso per cápita familiar, clima educativo y recepción de una asistencia monetaria estatal directa); y modelo 3: (región, quintil de ingreso per cápita familiar, clima educativo, recepción de una asistencia monetaria estatal directa, presencia de adultos mayores, presencia de menores de 14 años y sexo de la jefatura del hogar). Los valores de referencia utilizados se encuentran señalados en cada variable.

Coeficiente de regresión lineal= Indica cuántos gramos promedio diarios por adulto equivalente aumenta 0 disminuye el consumo aparente de frutas frescas ante la presencia de cada categoría de las variables independientes 0 el incremento de una unidad en la variable quintil de ingreso per cápita del hogar. Coeficiente de regresión estandarizado= Permite dar cuenta de la importancia relativa de las distintas variables en el modelo. GBA= Gran Buenos Aires; NOA= Noroeste Argentino; NEA=Nordeste Argentino.

${ }^{1}$ Los quintiles de ingreso fueron introducidos como variable cuantitativa.

${ }^{*}$ Resultados estadísticamente significativos: $p<0,05$.

**Resultados estadísticamente significativos: $p<0,01$. 
el modelo 2, todas tienen un efecto significativo sobre el consumo de frutas frescas. En cuanto al ingreso per cápita del hogar se observa que, a medida que aumenta el quintil de ingreso, se incrementa el consumo de frutas. También los hogares que reciben una asistencia económica directa del Estado consumen menos frutas por adulto equivalente que los que no reciben asistencia. Por último, cabe señalar que los hogares con un clima educativo alto consumen más frutas que los hogares con climas educativos bajos y medios.

En el modelo 3, se introducen las variables de composición del hogar, que producen un aumento en la bondad de ajuste del modelo (el $R^{2}$ pasa de 0,057 a 0,093 ), pero además modifican la magnitud del efecto que generan las variables socioeconómicas. Comenzando por el efecto de las variables de composición del hogar, se observa que los hogares con adultos mayores, sin menores de 14 años y con jefatura femenina consumen más frutas que los hogares sin adultos mayores, con menores de 14 años y con jefatura masculina. La presencia de adultos mayores es la variable con mayor importancia relativa en el modelo (el coeficiente de regresión estandarizado es 0,163 ).

En cuanto al efecto que genera la incorporación de las variables de composición del hogar sobre las variables socioeconómicas, se observa que cobrar una asistencia monetaria directa por parte del Estado pierde significación estadística. Por su parte, las diferencias entre los quintiles de ingreso continúan siendo significativas, pero disminuyen considerablemente. También disminuye la importancia relativa de la variable (el coeficiente de regresión estandarizado pasa de 0,197 en el modelo 2 a 0,139 en el modelo 3). En cambio, las diferencias de consumo según clima educativo del hogar aumentan y siguen una relación lineal, de forma tal que a menor clima educativo menor consumo de frutas. Los hogares con clima educativo bajo consumen 45,4 g menos que los de clima educativo alto, mientras que los hogares con clima educativo medio consumen $33,1 \mathrm{gr}$ diarios menos. También aumenta la relevancia de la variable en el modelo (en el caso de la variable dummy clima educativo bajo, el coeficiente de regresión estandarizado pasa de -0,062 en el modelo 2 a -0,151 en el modelo 3). Estos cambios en las variables socioeconómicas se explican porque en los hogares que no cobran una asistencia económica directa por parte del Estado y en los

Tabla 4. Consumo aparente diario de verduras frescas por adulto equivalente (en gramos) en hogares de zonas urbanas, según variables seleccionadas. Argentina, 2017-2018.

\begin{tabular}{|c|c|}
\hline Variables & $\begin{array}{l}\text { CAD de verduras } \\
\text { frescas (en } \\
\text { gramos) }\end{array}$ \\
\hline
\end{tabular}

\section{Región}

GBA

120,7

Pampeana

129,1

NOA

151,7

\begin{tabular}{l|l} 
NEA & 90,4
\end{tabular}

\begin{tabular}{ll} 
Cuyo 164,1 \\
\hline
\end{tabular}

\begin{tabular}{l|l} 
Patagonia 119,0 \\
\hline
\end{tabular}

Recepción de una asistencia monetaria estatal directa

Sin asistencia $\quad 137,3$

Con asistencia

Clima educativo del hogar

Alto $\quad 149,2$

\begin{tabular}{l|l} 
Medio 126,1 \\
\hline
\end{tabular}

Bajo

122,2

Quintil de ingreso per cápita del hogar (total del país)

\begin{tabular}{|l|r|}
\hline 1er quintil & 83,2 \\
\hline 2do quintil & 109,1 \\
\hline 3er quintil & 127,4 \\
\hline 4to quintil & 146,7 \\
\hline 5to quintil & 167,0 \\
\hline
\end{tabular}

Presencia de menores de 14 años en el hogar

\begin{tabular}{ll} 
NNo $\quad 154,9$ \\
\hline
\end{tabular}

Sí $\quad 87,0$

Presencia de adultos mayores en el hogar

$\begin{array}{ll}\text { NNo } & 109,1\end{array}$

Sí 173,0

Sexo de la jefatura del hogar

$\begin{array}{ll}\text { Masculino } \quad 117,2 & \end{array}$

\begin{tabular}{l|l|l} 
Femenino & 139,4
\end{tabular}

Fuente: Elaboración propia con base en la Encuesta Nacional de Gastos de Hogares 2017-2018.

$\mathrm{GBA}=$ Gran Buenos Aires; NOA= Noroeste Argentino; NEA=Nordeste Argentino. $C A D=$ Consumo aparente diario. 
quintiles de mayores ingresos tienen un mayor peso los hogares con adultos mayores $y$ sin menores de 14 años. Por su parte, en los hogares que tienen un clima educativo bajo, tienen un mayor peso los hogares con adultos mayores. $\mathrm{Y}$ como se vio anteriormente, los hogares con adultos mayores y que no tienen menores consumen más frutas por adulto equivalente.

\section{Consumo de verduras frescas}

En la Tabla 4 se observa el consumo diario por adulto equivalente de verduras frescas, cuyo promedio es de 126,7 g por día. En Cuyo $(164,1 \mathrm{~g})$ y en el NOA $(157,1 \mathrm{~g})$ es donde más se consume, mientras que es en el NEA $(90,4 \mathrm{~g})$ donde menos se consume. En cuanto al clima educativo del hogar, se observa que los hogares de clima educativo alto $(149,2 \mathrm{~g})$ son los que más consumen, y los de bajo $(122,2 \mathrm{~g})$ los que menos consumen. Con relación al quintil de ingreso per cápita del hogar, a medida que aumenta el quintil se incrementa el consumo de verduras por adulto equivalente. Entre los extremos se observa que los hogares del 5to quintil consumen el doble $(167,0 \mathrm{~g})$ que los del 1er quintil $(83,2 \mathrm{~g})$. A su vez, es mayor el consumo entre los hogares que no cobran una asistencia estatal monetaria directa $(137,3$ g) con relación a los hogares que lo cobran $(80,3$ g). Por último, con relación a las variables de composición del hogar se observa que es mayor el consumo en los hogares de jefatura femenina $(139,4 \mathrm{~g})$, que no tienen menores de 14 años $(154,9$ g) y que tienen adultos mayores $(173,0 \mathrm{~g})$ en comparación con los hogares de jefatura masculina $(117,2$ g), que tienen menores de 14 años $(87,0 \mathrm{~g})$ y que no tiene adultos mayores $(109,1 \mathrm{~g})$.

En la Tabla 5 se presentan los resultados de la regresión lineal múltiple para el consumo de verduras frescas. Comenzando por la región de residencia, se observa que, con relación al GBA, los hogares de Cuyo, del NOA y de la región Pampeana tienen un mayor consumo, los del NEA tienen un menor consumo y no hay diferencias significativas con los de la Patagonia. Esta relación tiende a mantenerse en todos los modelos, aunque se destaca que en el modelo 2, al incorporar las variables socioeconómicas, aumentan las diferencias con los hogares del NOA y del Cuyo (mayor consumo que los del GBA) y dejan de ser significativas las diferencias con los hogares del NEA (menor consumo que los del GBA, que vuelven a ser significativas en el modelo 3 al incorporar las variables de composición del hogar).

Con relación a las variables socioeconómicas, en el modelo 2 de la Tabla 5 (verduras frescas) se observan resultados similares a los del modelo 2 de la Tabla 3 (frutas frescas) para el ingreso y la recepción de asistencia monetaria estatal directa, pero no así para el clima educativo. En cuanto al quintil de ingreso per cápita del hogar, se observa que a medida que aumenta el quintil de ingreso aumenta el consumo de verduras frescas, y que en los hogares que reciben una asistencia monetaria directa del Estado consumen menos verduras por adulto equivalente. Por último, se destaca como llamativo que los hogares con un clima educativo alto consumen menos verduras frescas que los hogares con clima educativo bajo. En cambio, no hay diferencias significativas con quienes tienen nivel educativo medio.

En el modelo 3, al introducir las variables de composición del hogar, se observa que al igual que para el consumo de frutas, las diferencias entre los quintiles de ingreso continúan siendo estadísticamente significativas, pero disminuyen. Ello se aprecia en que se reduce el coeficiente de regresión lineal de 20,4 en el modelo 2 a 13,6 en el modelo 3 (indica cuánto aumenta el consumo de verduras frescas al aumentar un quintil de ingreso). $Y$ a su vez disminuye el coeficiente de regresión estandarizado (pasa de 0,163 a 0,109 ), aunque continúa siendo la variable con la mayor importancia relativa del modelo (similar a la presencia de adultos mayores cuyo coeficiente de regresión estandarizado es 0,103$)$. También se reducen las diferencias entre los hogares que reciben y los que no reciben una asistencia económica directa del Estado, sin embargo, estas continúan siendo estadísticamente significativas (mientras que en el consumo de fruta dejaron 
Tabla 5. Regresión lineal múltiple del consumo aparente diario de verduras frescas por adulto equivalente (en gramos), en hogares de zonas urbanas. Argentina, 2017-1818.

\begin{tabular}{|c|c|c|c|c|c|c|}
\hline & \multicolumn{2}{|c|}{ Modelo 1} & \multicolumn{2}{|c|}{ Modelo 2} & \multicolumn{2}{|c|}{ Modelo 3} \\
\hline & $\begin{array}{l}\text { Coeficiente } \\
\text { de regresión } \\
\text { lineal }\end{array}$ & $\begin{array}{c}\text { Coeficiente } \\
\text { de regresión } \\
\text { estandarizado }\end{array}$ & $\begin{array}{l}\text { Coeficiente } \\
\text { de regresión } \\
\text { lineal }\end{array}$ & $\begin{array}{c}\text { Coeficiente } \\
\text { de regresión } \\
\text { estandarizado }\end{array}$ & $\begin{array}{l}\text { Coeficiente } \\
\text { de regresión } \\
\text { lineal }\end{array}$ & $\begin{array}{c}\text { Coeficiente } \\
\text { de regresión } \\
\text { estandarizado }\end{array}$ \\
\hline \multicolumn{7}{|l|}{ Región } \\
\hline GBA (referencia) & - & - & - & - & - & - \\
\hline Pampeana & $8,4^{* *}$ & 0,023 & $8,5^{* *}$ & 0,023 & $6,6^{*}$ & 0,018 \\
\hline $\mathrm{NOA}$ & $31,0^{* *}$ & 0,051 & $49,3^{* *}$ & 0,081 & $47,1^{* *}$ & 0,077 \\
\hline NEA & $-30,3^{* *}$ & $-0,045$ & $-8,6$ & $-0,013$ & $-10,7^{*}$ & $-0,016$ \\
\hline Cuyo & $43,4^{* *}$ & 0,059 & $53,3^{* *}$ & 0,073 & $52,2^{* *}$ & 0,071 \\
\hline Patagonia & $-1,8$ & $-0,002$ & $-8,3$ & $-0,011$ & $-0,5$ & $-0,001$ \\
\hline \multicolumn{7}{|c|}{ Recepción de una asistencia monetaria estatal directa } \\
\hline Sin asistencia (referencia) & - & - & - & - & - & - \\
\hline Con asistencia & - & - & $-31,9 * *$ & $-0,070$ & $-12,5^{* *}$ & $-0,025$ \\
\hline \multicolumn{7}{|l|}{ Clima educativo del hogar } \\
\hline Alto (referencia) & - & - & - & - & - & - \\
\hline Medio & - & - & 0,2 & 0,000 & $-9,4^{*}$ & $-0,026$ \\
\hline Bajo & - & - & $17,3^{* *}$ & 0,049 & $-8,2$ & $-0,023$ \\
\hline \multicolumn{7}{|c|}{ Quintil de ingreso per cápita del hogar (total país) } \\
\hline Quintiles de ingreso $0^{1}$ & & & $20,4^{* *}$ & 0,163 & $13,6^{* *}$ & 0,109 \\
\hline \multicolumn{7}{|c|}{ Presencia de menores de 14 años en el hogar } \\
\hline № (referencia) & - & - & - & - & - & - \\
\hline Sí & - & - & - & - & $-37,0^{* *}$ & $-0,103$ \\
\hline \multicolumn{7}{|c|}{ Presencia de adultos mayores en el hogar } \\
\hline № (referencia) & - & - & - & - & - & - \\
\hline Sí & - & - & - & - & $40,6^{* *}$ & 0,103 \\
\hline \multicolumn{7}{|l|}{ Sexo de la jefatura del hogar } \\
\hline Masculina (referencia) & - & - & - & - & - & - \\
\hline Femenina & - & - & - & - & $20,8^{* *}$ & 0,058 \\
\hline Constante (ordenada al origen) & $120,7^{* *}$ & & $54,1^{* *}$ & & $82,1^{* *}$ & \\
\hline $\mathrm{R} 2$ & \multicolumn{2}{|c|}{0,008} & \multicolumn{2}{|c|}{0,043} & \multicolumn{2}{|c|}{0,07} \\
\hline
\end{tabular}

Fuente: Elaboración propia con base en la Encuesta Nacional de Gastos de Hogares 2017-2018.

Nota: Para la regresión lineal múltiple se utilizó el método de inclusión Intro y las variables independientes fueron incluidas de la siguiente forma: modelo 1 (región); modelo 2: (región, quintil de ingreso per cápita familiar, clima educativo y recepción de una asistencia monetaria estatal directa); y modelo 3: (región, quintil de ingreso per cápita familiar, clima educativo, recepción de una asistencia monetaria estatal directa, presencia de adultos mayores, presencia de menores de 14 años y sexo de la jefatura del hogar). Los valores de referencia utilizados se encuentran señalados en cada variable.

Coeficiente de regresión lineal= Indica cuántos gramos promedio diarios por adulto equivalente aumenta o disminuye el consumo aparente de frutas frescas ante la presencia de cada categoría de las variables independientes o el incremento de una unidad en la variable quintil de ingreso per cápita del hogar. Coeficiente de regresión estandarizad $0=$ Permite dar cuenta de la importancia relativa de las distintas variables en el modelo. GBA= Gran Buenos Aires; NOA= Noroeste Argentino; NEA=Nordeste Argentino.

'Los quintiles de ingreso fueron introducidos como variable cuantitativa.

*Resultados estadísticamente significativos: $p<0,05$.

**Resultados estadísticamente significativos: $p<0,01$. 
de serlo en el modelo 3). Por último, los hogares de clima educativo alto consumen más verduras frescas que los de clima educativo medio, mientras que las diferencias con los de clima educativo bajo no son estadísticamente significativas.

En cuanto a las variables de composición del hogar, en el modelo 3, una vez controlados los efectos de las variables socioeconómicas y de la región, se observa que los hogares con adultos mayores consumen más verdura por adulto equivalente que los que no tienen adultos mayores, que los hogares sin menores de 14 años consumen más que los que tienen menores y que los hogares con jefatura femenina consumen más que los de jefatura masculina. Se destaca que el efecto de las variables de composición del hogar para el consumo de verduras frescas es similar al del consumo de frutas frescas.

\section{DISCUSIÓN}

El objetivo de este trabajo fue analizar la incidencia de diferentes factores sociales, económicos, geográficos y de composición del hogar en el consumo aparente de verduras y frutas de la población urbana de la Argentina. Los resultados muestran, en primer lugar, que el consumo promedio de verduras y de frutas frescas es muy bajo y está lejos de alcanzar los valores recomendados por la OMS y más aún por las Guías Alimentarias para la Población Argentina. Este bajo consumo es concordante con lo observado recientemente en la ENNyS 2018 ${ }^{(16)}$, y con la ENFR $2018^{(15)}$. Además, según los análisis de tendencias realizados con datos de la ENGHo, el consumo aparente de frutas y verduras es decreciente, con un descenso del $41 \%$ para las frutas y del $13 \%$ para las verduras entre 1996 y $2013^{(13)}$. Los resultados de este trabajo muestran que continúa la tendencia decreciente para el consumo aparente de frutas frescas, que descendió un $7 \%$ con relación al relevamiento del 2012-2013. En cambio, el de verduras frescas tuvo un aumento del $6 \%$ con relación a 2012-2013, aunque continúa siendo muy inferior a los resultados de 1996-1997(13) y a los valores recomendados ${ }^{(10,12)}$.

En segundo lugar, el análisis bivariado arroja que el consumo aumenta en función al ingreso del hogar. Resultados similares se encontraron en trabajos que utilizaron datos de la encuesta de gastos de hogares para analizar el consumo aparente de frutas y verduras ${ }^{(45,46)}$, así como de otros alimen$\operatorname{tos}^{(17,19,47,48,49,50,51)}$. En este sentido, destacamos como un aporte del presente trabajo haber incorporado en el análisis otras variables para comprender los determinantes del consumo aparente. Con relación a otras variables socioeconómicas, el consumo también fue más bajo en los hogares con menor clima educativo y en aquellos con recepción de una asistencia monetaria estatal directa. Estos datos concuerdan con la ENNyS 2018, que evidenció que el consumo diario de frutas y verduras fue significativamente menor en los grupos con bajo nivel educativo y de menores ingresos ${ }^{(16)}$. En relación con la asistencia estatal, las evaluaciones disponibles sobre la compra de alimentos con la Tarjeta Alimentar, programa nacional implementado a partir de 2020 , dan cuenta que el $82,1 \%$ de los hogares con niños y niñas de 0 a 6 años beneficiarios declararon haber adquirido frutas $y$ verduras en su última compra de alimentos, sin diferencias significativas con el total de hogares con niños y niñas ${ }^{(52)}$, destacando que no se reportan datos sobre la cantidad adquirida. Sin embargo, dicho estudio indica que, entre los hogares vulnerables, aquellos que reciben la Tarjeta Alimentar compran más frutas y verduras que los que no la reciben, lo que indica la importancia de evaluar el impacto diferencial de las distintas políticas de asistencia alimentaria considerando la composición y las características del hogar ${ }^{(53)}$.

Según las variables de composición del hogar, se observó menor consumo en hogares sin adultos mayores, con menores de 14 años y con jefatura masculina. Los resultados están en línea con estudios previos que marcan el rol central de las mujeres en las decisiones alimentarias de los hogares, los cambios en las pautas de consumo hacia una dieta con mayor contenido de frutas y verduras que 
suelen acompañar el proceso de envejecimiento $^{(29,34,35)}$, y el papel de las preferencias de niños y adolescentes en las decisiones de compra de alimentos ${ }^{(31,36)}$. En cuanto a las regiones geográficas, el menor consumo de frutas frescas y verduras frescas se observó en hogares del NEA y de la Patagonia mientras que, en la ENNyS 2018, el NEA junto con el NOA y Cuyo presentaron resultados más desfavorables en consumo diario de frutas. Por su parte, los resultados son diferentes para las verduras dado que, en la ENNyS 2018, el NEA presentó la mayor frecuencia de consumo diario y Cuyo la menor ${ }^{(16)}$.

En tercer lugar, el análisis multivariado permitió evidenciar el efecto de cada variable independiente controlando las restantes. En este sentido, en cuanto a las variables socioeconómicas, se observó que el efecto del ingreso disminuye considerablemente una vez controladas las variables de composición del hogar. Por su parte, los hogares con clima educativo alto son los que presentaron mayor consumo aparente de frutas, diferencias que aumentan una vez controlados los efectos de la composición del hogar. En cambio, en el consumo aparente de verduras frescas no hay diferencias estadísticamente significativas con los hogares de bajo clima educativo una vez controlado el efecto del resto de las variables socioeconómicas, geográficas y las de composición del hogar. En tanto que los hogares que reciben asistencia monetaria estatal directa tienen un menor consumo aparente de ambos grupos de alimentos, pero una vez controlado el efecto del resto de las variables independientes estas diferencias dejan de ser estadísticamente significativas para el consumo aparente de frutas y disminuyen considerablemente para el de verduras.

Los resultados observados en relación con la composición del hogar, continúan siendo estadísticamente significativos una vez controladas el resto de las variables consideradas en el estudio. Cabe resaltar el fuerte efecto que tiene la presencia de adultos mayores, al punto de ser la variable que mayor relevancia tiene en el consumo aparente de frutas frescas y una de las de mayor importancia en el de verduras frescas.
En relación con las diferencias geográficas, se destaca que el bajo consumo aparente tanto de frutas como de verduras en la región del NEA, se mantienen incluso una vez controlados los efectos de las variables socioeconómicas y de composición del hogar. Como contrapartida, cabe señalar que, si bien el NOA y Cuyo presentaron un menor consumo de frutas, una vez controlados los efectos de las variables socioeconómicas y de composición del hogar, las diferencias con el GBA dejan de ser estadísticamente significativas.

Los hallazgos de nuestro estudio permiten evidenciar la importancia de incorporar variables de la composición del hogar junto con las estructurales que suelen ser consideraras como más relevantes y como condicionantes principales del consumo de alimentos como, por ejemplo, el ingreso o el nivel educativo. Cabe destacar también la utilidad de los modelos de análisis multivariado, ya que como se evidenció en el análisis, al controlarse los efectos de las variables de composición del hogar se pueden modificar los efectos de las variables estructurales.

Es fundamental pensar los resultados obtenidos en este trabajo desde la perspectiva de los sistemas alimentarios y su indisociable vínculo con la salud de la población ${ }^{(54)}$. Nuestra salud y bienestar se ven directamente afectados por los sistemas alimentarios que determinan la forma de cultivar, cosechar, elaborar, transportar, comercializar, consumir y desechar alimentos, a través de múltiples vías interrelacionadas, que se han visto afectadas por la pandemia de Covid-19 exacerbando las desigualdades sociales ${ }^{(55)}$. En relación con la producción de alimentos en la Argentina, según los datos del Censo Nacional Agropecuario 2018, del total de la superficie implantada, solo se dedica el 1,4\% (490.770 hectáreas) a la producción de cultivos frutales y el 0,4\% (139.585 hectáreas) a la de hortalizas ${ }^{(56)}$. Si bien se estima que la producción bruta es superior al consumo recomendable per cápita, el 50\% tiene como destino la industria y un $10 \%$ la exportación, además del importante porcentaje que se pierde y desperdicia durante la producción, el transporte, la comercialización y el consumo de alimentos. 
Los muy bajos porcentajes de superficie destinados a la producción de frutas y hortalizas se originan en el desplazamiento de determinados cultivos debido a la expansión del agronegocio, en el marco de la globalización de la cadena agroalimentaria ${ }^{(22,57)}$.

A pesar de lo anterior, los datos de las Hojas de Balance de Alimentos de la FAO muestran que la disponibilidad per cápita de frutas y verduras en la Argentina es, en promedio, $438 \pm 37 \mathrm{~g} / \mathrm{d}$ entre 1961 y 2018 , con valores relativamente estables en el tiempo que apenas alcanzan en algunos periodos a la recomendación mínima de la OMS $(400 \mathrm{~g} / \mathrm{d})^{(58)}$. En cuanto al tipo de comercialización, se destaca que la comercialización indirecta (vía un mercado concentrador o circuito de gran distribución como supermercados o hipermercados) es mucho mayor a la directa (del productor al minorista o al de los sistemas alimentarios y su indisociable vínculo con la salud de la población consumidora). En el caso de las hortalizas, la comercialización indirecta se estima que es cuatro veces mayor que la directa ${ }^{(59)}$.

Asimismo, en la Argentina, las políticas de control de precios como "Precios esenciales" y "Precios cuidados" del Ministerio de Desarrollo Productivo, han formado parte de las medidas en contextos de inflación de los últimos años, pero ambas políticas han sido delineadas desde la lógica económica del consumo y no desde las recomendaciones nutricionales y de salud, y el listado de alimentos apenas incluye 4 o 5 verduras y frutas dentro los ítems contemplados ${ }^{(60,61)}$. En este sentido, cabe destacar que luego del período de estudio (2017-2018) se continuó con un importante proceso inflacionario en el que los precios de los alimentos, y en particular de las frutas y de las verduras, estuvieron por encima del incremento general del índice de precios al consumidor. Según los últimos datos disponibles del Instituto Nacional de Estadística y Censos (INDEC), tomando como referencia diciembre de 2016, en octubre de 2021 el índice de precios al consumidor tuvo un aumento del $436,5 \%$, mientras que los alimentos tuvieron un aumento de $478,2 \%$, las frutas de $475,9 \%$ y las verduras, hortalizas y legumbres un $519,6 \%{ }^{(20)}$.
La situación nutricional de la población representa el último eslabón del sistema alimentario, la malnutrición se relaciona con la producción, disponibilidad, acceso y comportamientos de consumo de alimentos ${ }^{(62)}$. La Argentina presenta alta prevalencia de exceso de peso y anemia, prevalencia moderada de retraso en el crecimiento y baja frecuencia de emaciación y bajo peso. Todas las formas de malnutrición muestran una fuerte relación con la desigualdad socioeconómica y educativa $^{(63)}$. Existe consenso entre varios autores, que atribuyen una relación directa entre la malnutrición en contextos de pobreza con la accesibilidad económica a los alimentos ${ }^{(64)}$.

Por su parte, el marketing y la publicidad de alimentos se orientan mayormente a los alimentos de baja calidad nutricional, como ha sido evidenciado en varios estudios locales ${ }^{(65,66)}$, impactando en las decisiones de compra de los hogares en detrimento de alimentos más saludables, especialmente en los hogares con niños, niñas y adolescentes ${ }^{(36)}$.

Desde el punto de vista metodológico, por la escasez de datos de consumo alimentario obtenidos a través de metodologías de recolección individual y con representatividad nacional, las encuestas de gastos de hogares proporcionan una fuente promisoria de información ${ }^{(67)}$. Han pasado de realizarse en apenas 22 países en $1950^{(68)}$ a más de 100 en la actualidad ${ }^{(69)}$, en muchos casos, proporcionando información periódica y seriada de muestras en entornos urbanos y rurales con muy buena representatividad. Además, la forma de obtener información alimentaria en los hogares es menos intrusiva que otros métodos. Sin embargo, el uso de la compra de alimentos como estimación del consumo aparente plantea limitaciones intrínsecas, como la ausencia de datos sobre la distribución de alimentos entre los diferentes miembros del hogar y la falta de registro de desperdicios de alimentos o aquellos destinados para consumo animal, y de los alimentos que se reciben por programas sociales o gratuitamente. A pesar de las limitaciones, las encuestas de gastos de hogares constituyen una herramienta subutilizada, pero con un gran potencial para evaluar el consumo de alimentos y bebidas, sobre todo en los países 
de bajos y medianos ingresos ${ }^{(70)}$. La información obtenida puede ser de mayor utilidad en familias con bajos ingresos, donde los desperdicios u otros destinos de los alimentos adquiridos son mínimos. Otra limitación relativa al análisis es que, al utilizar la variable región, el nivel de agregación no permite dar cuenta de la heterogeneidad que presentan las diferentes zonas dentro de una misma región.

Una última limitación de nuestro estudio es que seguimos un enfoque cuantitativo basado en el análisis de datos secundarios de encuesta. Las prácticas alimentarias, como fenómeno sociocultural, revisten una gran complejidad que puede captarse limitadamente a través de una encuesta. Las metodologías cuantitativas hacen un recorte de la realidad en términos de variables y sus relaciones. $\mathrm{Si}$ bien esta forma de abordar un fenómeno social conlleva una simplificación de la riqueza y profundidad en el análisis, en el caso de una encuesta con una muestra estadísticamente representativa de cobertura nacional como la ENGHo, se gana en la posibilidad de realizar generalizaciones estadísticas de los patrones de asociación observados entre las variables analizadas y las desigualdades sociales y aspectos relativos a la organización del hogar. Consideramos que los estudios cuantitativos sobre consumo alimentario, con sus limitaciones y aportes, indudablemente complementan los abordajes cualitativos antropológicos y sociológicos que permiten profundizar con una aproximación holística y situada las decisiones de consumo y las prácticas alimentarias en los hogares, sus condiciones de vida y los significados que portan para sus integrantes.

Entre las fortalezas de nuestro estudio, se destacan las relativas a la fuente de información, como las que se han mencionado con

\section{FINANCIAMIENTO}

El trabajo se hizo con el apoyo del Proyecto Ciencia y Tecnología contra el Hambre 2020-C105 “Desigualdades sociales y alimentación: relaciones entre calidad de la alimentación, condiciones de vida y composición de los hogares argentinos" del Ministerio de Ciencia Tecnología e Innovación de Argentina, y del Proyecto PICT 2019-03036 “Desigualdades sociales en los patrones de actividad física y alimentación de la población adulta relación al tamaño de la muestra y su representatividad en localidades de al menos 2.000 habitantes. Respecto del tipo de análisis, este es el primer estudio que utiliza modelos de regresiones múltiples para evaluar el consumo aparente de frutas y verduras a partir de datos de la ENGHo. En relación con la perspectiva analítica, este es el primer trabajo que incluye en simultáneo variables socioeconómicas, geográficas y de composición de los hogares. Existe un antecedente que utilizó regresión lineal múltiple con datos de frecuencia de consumo de frutas y verduras tomando como fuente la ENFR 2013 y con otra selección de variables explicativas (como edad, ingreso, nivel educativo, actividad física, exceso de peso y consumo de alcohol) $)^{(71)}$.

\section{CONCLUSIÓN}

Este análisis evidencia el bajo consumo de frutas y verduras en la población argentina, y la influencia de las condiciones de vida y composición de los hogares sobre el consumo. Destacando la ausencia de adultos mayores, la presencia de menores de 14 años y la jefatura masculina como factores asociados al menor consumo de frutas y verduras en los hogares argentinos.

Los hallazgos de este trabajo destacan la importancia de conocer las desigualdades sociales asociadas al consumo de alimentos recomendados para una alimentación saludable, dado que contribuyen a planificar acciones y políticas focalizadas en pos de garantizar la seguridad alimentaria y nutricional y mejorar la calidad de la alimentación de la población, especialmente de los grupos más vulnerados.

y los hogares urbanos de Argentina, 2014-2018"de la Agencia Nacional de Promoción de la Investigación, el Desarrollo Tecnológico y la Innovación.

\section{CONFLICTO DE INTERESES}

Las autoras declaran no tener vínculos o compromisos que condicionen lo expresado en el texto y que puedan ser entendidos como conflicto de intereses. 


\section{REFERENCIAS BIBLIOGRÁFICAS}

1. Fanzo J, Drewnowski A, Blumberg J, Miller G, Kraemer K, Kennedy E. Nutrients, foods, diets, people: Promoting healthy eating. Current Developments in Nutrition. 2020;4(6):nzaa069.

2. Rabe Cáez Ramírez G, Casas Forero N. Formar en un estilo de vida saludable: otro reto para la ingeniería y la industria. Educación y Educadores. 2007; 10:103-117.

3. Schwingshackl L, Schwedhelm C, Hoffmann G, Lampousi AM, Knuppel S, Iqbal K, et al. Food groups and risk of all-cause mortality: a systematic review and metaanalysis of prospective studies. The American Journal of Clinical Nutrition. 2017;105(6):1462-1473.

4. Schwingshackl L, Schwedhelm C, Hoffmann G, Knuppel S, Laure Preterre A, Iqbal K, et al. Food groups and risk of colorectal cancer. International Journal of Cancer. 2018;142(9):1748-1758

5. Schwingshackl L, Schwedhelm C, Hoffmann G, Knuppel S, Iqbal K, Andriolo $\mathrm{V}$, et al. Food groups and risk of hypertension: A systematic review and dose-response meta-analysis of prospective studies. Advances in Nutrition. 2017;8(6):793-803.

6. Schwingshackl L, Hoffmann G, Lampousi AM, Knuppel S, Iqbal K, Schwedhelm C, et al. Food groups and risk of type 2 diabetes mellitus: a systematic review and metaanalysis of prospective studies. European Journal of Epidemiology. 2017;32(5):363-375.

7. Collaborators GBDD. Health effects of dietary risks in 195 countries, 1990-2017: a systematic analysis for the Global Burden of Disease Study 2017. The Lancet 2019;393(10184):1958-1972.

8. Popkin BM. The nutrition transition and obesity in the developing world. The Journal of Nutrition. 2001;131(3):S871-S873.

9. Popkin BM, Gordon-Larsen P. The nutrition transition worldwide obesity dynamics and their determinants. International Journal of Obesity and Related Metabolic Disorders. 2004;28(Suppl 3):S2-S9.

10. Organización Mundial de la Salud. Dieta, nutrición y prevención de enfermedades crónicas. Ginebra: OMS, FAO; 2003.

11. Wolfenden L, Barnes C, Lane C, McCrabb S, Brown HM, Gerritsen S, et al. Consolidating evidence on the effectiveness of interventions promoting fruit and vegetable consumption: an umbrella review. International Journal of Behavioral Nutrition and Physical Activity. 2021;18(1):11.

12. Argentina, Ministerio de Salud. Guías Alimentarias para la Población Argentina. Buenos Aires: Ministerio de Salud; 2016.

13. Zapata M, Rovirosa A, Carmuega E. Cambios en el patrón de consumo de alimentos y bebidas en Argentina, 1996-2013. Salud Colectiva. 2016;12(4):473-486.
14. Zapata ME, Rovirosa A, Carmuega E. La mesa argentina en las últimas dos décadas. Cambios en el patrón de consumo de alimentos y nutrientes (1996-2013). Ciudad Autónoma de Buenos Aires: CESNI; 2016.

15. Argentina, Ministerio de Salud y Desarrollo Social. 4ta Encuesta Nacional de Factores de Riesgo: Informe definitivo [Internet]. 2019 [citado 1 feb 2021]. Disponible en: https://tinyurl.com/2p88ad53.

16. Argentina, Ministerio de Salud y Desarrollo Social. $2^{\circ}$ Encuesta Nacional de Nutrición y Salud: Principales resultados de los indicadores priorizados. Resumen Ejecutivo. 2019 Acceso el 6 de abril de 2021; Disponible en: https://tinyurl.com/2p96mbxy.

17. Aguirre P. La comida en los tiempos del ajuste. En Torrado S, (comp.). El costo social del ajuste (Argentina 1976-2002). Buenos Aires: Editorial Edhasa: 2009. p. 52102.

18. Salvia A. Desarrollo humano e integración social en la Argentina urbana 2010-2016: Documento estadístico. Buenos Aires: UCA; 2017.

19. Aguirre P. Estrategias de consumo: qué comen los argentinos que comen. Buenos Aires: Miño y Dávila; 2005.

20. Instituto Nacional de Estadísticas y Censos. Índices y variaciones porcentuales mensuales e interanuales según principales aperturas de la canasta: Diciembre de 2016-octubre de 2021 [Internet]. 2021 [citado 1 feb 2021]. Disponible en: https://tinyurl.com/4bxv6dpb.

21. FAO, FIDA, OPS, WFP, UNICEF. América Latina y el Caribe: Panorama regional de la seguridad alimentaria y nutricional 2021: estadísticas y tendencias. Santiago: FAO; 2021.

22. Teubal M, Rodriguez J. Agro y Alimentos en la globalización: Una perspectiva crítica. Buenos Aires: Editorial La Colmena; 2002.

23. Alvarez MFS. Pocos ganan, muchos pierden: Soja, agroquímicos y salud. Villa María: Eduvim; 2009.

24. López Arellano O, Escudero JC, Carmona LD. Los determinantes sociales de la salud: Una perspectiva desde el Taller Latinoamericano de Determinantes Sociales de la Salud, ALAMES. Medicina Social. 2008;3(4):323-335.

25. Ortiz-Hernández L. La necesidad de un nuevo paradigma en el campo de la alimentación y nutrición. En: Jarillo Soto EG, Guinsberg E, (comps.). Temas y desafíos en salud colectiva. Buenos Aires: Lugar Editorial; 2007.

26. Breilh J. La epidemiología crítica: una nueva forma de mirar la salud en el espacio urbano. Salud Colectiva. 2010;6(1):83-101.

27. Aguirre P. Seguridad alimentaria: Una visión desde la antropología alimentaria. En: Sabulsky J, Ezpeleta ML, Chesta M. Enfoque integral del desarrollo en la infancia: el futuro comprometido. Córdoba: Fundación CLACYD; 2004 
28. Piaggio LR. El derecho a la alimentación en entornos obesogénicos: Reflexiones sobre el rol de los profesionales de la salud. Salud Colectiva. 2016;12(4):605-619.

29. Neuman N, Eli K, Nowicka P. Feeding the extended family: gender, generation, and socioeconomic disadvantage in food provision to children. Food, Culture \& Society. 2019;22(1):45-62.

30. Wei-ting C. "Junk food" to "treats": How poverty shapes family food practices. Food, Culture \& Society. 2016;19(1):151-170.

31. Freidin B. Alimentación y riesgos para la salud: visiones sobre la alimentación saludable y prácticas alimentarias de mujeres y varones de clase media en el Área Metropolitana de Buenos Aires. Salud Colectiva. 2016;12(4):519-536.

32. Koch S. A theory of grocery shopping: food,choice and conflict. London: Berg Publishers; 2012.

33. Zafra-Aparici E. Aprender a comer en casa: modelos de género y socialización alimentaria. En: Piaggio R, Solans AM, (coord.). Enfoques socioculturales de la alimentación: lecturas para el equipo de salud. Buenos Aires: Librería Akadia Editorial; 2014. p. 277-303.

34. Sydner YM, Fjellström C, Lumbers M, Sidenvall B, Raats M. Food habits and foodwork. Food, Culture \& Society. 2007;10(3):367-387.

35. Bukman AJ, Ronteltap A, Lebrun M. Interpersonal determinants of eating behaviours in Dutch older adults living independently: a qualitative study. BMC Nutrition. 2020;6(55).

36. Elliot C. Publicidad de alimentos para la diversión (fun foods): Descripción y análisis de los mensajes en los alimentos dirigidos a los niños/as en los supermercados. En: Piaggio LR, Solans AM, (cood.). Enfoques socioculturales de la alimentación: lecturas para el equipo de salud. Buenos Aires: Akadia; 2014.

37. Instituto Nacional de Tecnología Agropecuaria. Alimentos y cocinas regionales de America: Resumen del primer foro, 2017. Ciudad Autónoma de Buenos Aires: Ediciones INTA; 2018.

38. Diez Roux A, Mair C. Neighborhoods and health. Annals of the New York Academy of Sciences. 2010;1186(1):125-145.

39. Argentina, Ministerio de Salud, Subsecretaría de Estrategias Sanitarias. SARA: Sistema de Análisis y Registro de Alimentos, Versión 1.2.22 ed2007 [Internet] 2021 [citado 1 feb 2021]. Disponible en: https://datos.dinami. gov.ar/sara/

40. López L, Suárez M. Alimentación Saludable: Guía práctica para su realización. Buenos Aires: Hipocrético; 2012.

41. Instituto Nacional de Estadísticas y Censos. El gasto de Consumo de los Hogares Urbanos en la Argentina: Un análisis a partir de las mediciones de 1996/1997, 2004/2005 y 2012/2013. Ciudad Autónoma de Buenos Aires: INDEC; 2014
42. Instituto Nacional de Estadísticas y Censos. Encuesta Nacional de Gasto de Hogares 2017-2018: Informe de ingresos. Buenos Aires: INDEC; 2020.

43. López-Roldán P, Fachelli S. Metodología de la investigación social cuantitativa. Bellaterra: UAB; 2016.

44. Chitarroni H. El análisis de correlación y regresión lineal entre variables cuantitativas. Buenos Aires: USAL; 2002.

45. Bertollo M, Martire Y, Rovirosa A, Zapata ME. Patrones de consumo de alimentos y bebidas según los ingresos del hogar de acuerdo a los datos de la Encuesta Nacional de Gastos de los Hogares (ENGHo) del año 2012-2013. Diaeta (BAires). 2015;33(153):7-11.

46. Zapata ME, Rovirosa A, Carmuega E. Urbano y rural: diferencias en la alimentación de los hogares argentinos según nivel de ingreso y área de residencia. Salud Colectiva. 2019;15:39-40.

47. Arcidiácono M, Tortarol D. Medición de la pobreza en Argentina y sus regiones: el enfoque de Engel de kilocalorías [Internet]. 2013 [citado 1 feb 2021]. Disponible en: https://tinyurl.com/yba5e458.

48. Britos S, Saraví A, Vilella F. Buenas prácticas para una alimentación saludable de los argentinos [Internet]. 2010 [citado 1 feb 2021]. Disponible en: https://tinyurl. com/2zdbab44.

49. Britos S. La alimentación en tiempos de crisis: Intervenciones sociales en relación con los precios de alimentos. Archivos Argentinos de Pediatría. 2002;100(5):402-411.

50. Pace Guerrero I. Estimación de sistemas de ecuaciones de demanda para tipos de carnes en Argentina para el periodo 1996/97-2012/13. I Jornadas Nacionales de Econometría, Mar del Plata: Facultad de Ciencias Económicas y Sociales, Universidad Nacional de Mar del Plata; 2014.

51. Pace Guerrero I, Berges M, Casellas K. Estimaciones de elasticidades de demanda para carnes y pescado en Argentina. Anales de la Asociación Argentina de Economía Política [Internet]. 2014 [citado 1 feb 2021]. Disponible en: https://tinyurl.com/yckkf3he.

52. Tuñón I, Poy S, Salvia A. La Tarjeta Alimentar a un año de su implementación: Una caracterización sociodemográfica, socioalimentaria y socioeconómica de los hogares destinatarios. Ciudad Autónoma de Buenos Aires: Educa; 2021.

53. Poy S, Salvia A, Tuñón I. Evaluación de impacto del Programa Tarjeta ALIMENTAR. Efectos directos e indirectos en la inseguridad alimentaria, consumos alimentarios y no alimentarios. Ciudad Autónoma de Buenos Aires: Educa; 2021.

54. Organización de las Naciones Unidas para la Alimentación y la Agricultura. Frutas y verduras - esenciales en tu dieta. Año internacional de las Frutas y Verduras, 2021: Documento de antecedentes. Roma: FAO; 2020. 
55. Organización Mundial de la Salud. Sistemas alimentarios para una mejor salud. Resumen ejecutivo [Internet]. 2021 [citado 1 feb 2021]. Disponible en: https:// tinyurl.com/yc8m48hh.

56. Instituto Nacional de Estadística y Censos. Censo Nacional Agropecuario: Resultados Definitivos [Internet]. 2021 [citado 1 feb 2021]. Disponible en: https://tinyurl. com/5n6bjxxd.

57. Domínguez D. La soberanía alimentaria como enfoque crítico y orientación alternativa del sistema agroalimentario global. Pensamiento Americano. 2015;8(15):146-175.

58. Zapata M, Rovirosa A. La alimentación en la argentina: Una mirada desde distintas aproximaciones CAPA I, Disponibilidad de alimentos y nutrientes a nivel país [Internet]. Ciudad Autónoma de Buenos Aires: CESNI; 2021 [citado 1 feb 2021]. Disponible en: https://tinyurl. com/bde7c3m4

59. Giacobone G, Castronuovo L, Tiscornia V, Allemandi L. Análisis de la cadena de suministro de frutas y verduras en Argentina [Internet]. 2018 [citado 1 feb 2021]. Disponible en: https://tinyurl.com/2p94vusz.

60. Argentina, Ministerio de Desarrollo Productivo. Precios Cuidados [Internet]. 2021 [citado 1 feb 2021]. Disponible en: https://tinyurl.com/2dxzbyvf.

61. Argentina, Ministerio de Desarrollo Productivo, Secretaría de Comercio Interior. Resolución 100/2020 [Internet]. 2020 [citado 1 feb 2021]. Disponible en: https:// tinyurl.com/2vuuuyme.

62. Organización de las Naciones Unidas para la Alimentación y la Agricultura, Organización Panamericana de la Salud. Panorama de la seguridad alimentaria y nutricional en América Latina y el Caribe. Santiago: FAO, OPS; 2017.

63. Zapata ME, Soruco AI, Carmuega E. Malnutrition in all its forms and socio-economic indicators in Argentina. Public Health Nutrition. 2019;23(S1):S13-S20.
64. Abeyá Gilardón E. Una evaluación crítica de los programas alimentarios en Argentina. Salud Colectiva. 2016;12(4):589-604.

65. Gómez P, Zapata ME, Rovirosa A, Gotthelf S, Ferrante $\mathrm{D}$. Técnicas de marketing en publicidades de alimentos y bebidas en canales infantiles, diferencias según calidad nutricional. Revista Argentina de Salud Pública. 2017;8(33):22-27.

66. Gómez P, Tamburini C, Rodríguez García V, Chamorro $\mathrm{V}$, Carmuega E. Estrategias de marketing en sitios web de marcas de alimentos y bebidas consumidos por niños, niñas y adolescentes en la Argentina. Archivos Argentinos de Pediatría. 2021;119(1):51-55.

67. Sununtnasuka C, Fiedler JL. Can household-based food consumption surveys be used to make inferences about nutrient intakes and inadequacies? A Bangladesh case study. Food Policy. 2017;72:121-131.

68. Jolliffe D, Lanjouw P, Chen S, Kraay A, Meyer C, Negre $M$, et al. A measured approach to ending poverty and boosting shared prosperity: Concepts, data, and the twin goals. Washington DC: World Bank; 2014.

69. Ferreira FHG, Chen S, Narayan A, Sangraula P, Dabalen AL, Serajuddin $U$, et al. A global count of the extreme poor in 2012: Data issues, methodology and initial results. Policy Research Working Paper. Washington DC: The World Bank; 2015.

70. Fiedler JL, Lividini K, Bermudez OI, Smitz MF. Household consumption and expenditures surveys (HCES): a primer for food and nutrition analysts in low- and middle-income countries. Food and Nutrition Bulletin. 2012;33(3 Suppl):S170-S184.

71. Adrogué C, Orlicki ME. Factores relacionados al consumo de frutas y verduras en base a la Encuesta Nacional de Factores de Riesgo en Argentina. Revista Pilquen - Sección Ciencias Sociales. 2019;22(3):70-82.

\section{FORMA DE CITAR}

Ballesteros MS, Zapata ME, Freidin B, Tamburini C, Rovirosa A. Desigualdades sociales en el consumo de verduras y frutas según características de los hogares argentinos. Salud Colectiva. 2022;18:e3835. doi: 10.18294/sc.2022.3835.

Recibido: 12 oct 2021 | Versión final: 9 dic 2021 | Aprobado: 17 dic 2021 | Publicado en línea: 21 feb 2022

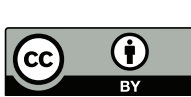

Esta obra está bajo una licencia Creative Commons Atribución 4.0 Internacional (CC BY 4.0). Atribución - Se debe dar crédito de manera adecuada, brindar un enlace a la licencia, e indicar si se han realizado cambios. Puede hacerlo en cualquier forma razonable, pero no de forma tal que sugiera que usted o su uso tienen e apoyo de la licenciante. Sin restricciones adicionales - No se pueden aplicar términos legales ni medidas tecnológicas que restrinjan legalmente a otras personas a hacer cualquier uso permitido por la licencia. 